\title{
Cross talk in phase-coded holographic memories
}

\author{
Kevin Curtis \\ Northrop B-2 Division, Pico Rivera, California 90660, and Department of Electrical Engineering, \\ California Institute of Technology, MS 116-81, Pasadena, California 91125
}

Demetri Psaltis

Department of Electrical Engineering, California Institute of Technology, MS 116-81, Pasadena, California 91125

Received June 14, 1993; revised manuscript received July 27, 1993; accepted August 2, 1993

\begin{abstract}
The cross talk between holograms multiplexed with Walsh-Hadamard phase codes is analyzed. Each hologram is stored with a reference beam that consists of $N$ phase-coded plane waves. The signal-to-noise ratio (SNR) is calculated for a recording schedule for which the center of each stored image coincides with the nulls of the selectivity function for the adjacent plane-wave components of the reference beam. The SNR characteristics for phase coding with Walsh-Hadamard phase codes are then compared with the SNR for angle and wavelength multiplexing.
\end{abstract}

\section{INTRODUCTION}

One can record multiple holograms by changing either the reference-beam angle ${ }^{1}(\theta$ multiplexing) or the recording wavelength ${ }^{2}$ ( $\lambda$ multiplexing) or by phase coding the reference beams ${ }^{3-5}$ ( $\phi$ multiplexing). The signal-to-noise ratio (SNR) of the recalled information determines the storage capability of holographic memories. One of the contributions to the noise is cross talk that is due to energy diffracted from non-Bragg-matched gratings. The analysis that we present here is analogous to the method used by Gu $e t a l .^{6}$ and by Curtis et al. ${ }^{7}$ This paper contains two main results. First, we show that each phase-coded hologram has almost the same SNR on reconstruction, once we exclude one bad code. This is in contrast to $\theta$ multiplexing, in which most holograms have much better SNR than the worst hologram, depending on their angular position with respect to the other holograms. Second, we show that the SNR for a given number of holograms is better for $\phi$ than for $\theta$ multiplexing.

\section{THEORY}

Fourier-transform holograms are multiplexed in a volume holographic medium with the setup shown in Fig. 1. The $m$ th hologram is formed by interfering the signal with a reference beam $R_{m}$ that consists of $N$ plane-wave components, with the phase of the $i$ th component being modulated by $P_{i}{ }^{m}$ :

$$
R_{m}=\sum_{i=-N / 2}^{N / 2-1} P_{i}^{m} \exp \left(i \mathbf{k}_{i} \cdot \mathbf{r}\right)
$$

In this paper we assume that the $P_{i}^{m}$ 's are either 1 or -1 and that they are the Walsh-Hadamard codes, which are orthogonal and easy to construct. The method used for constructing them is given in Ref. 8 . This reference beam interferes inside the holographic medium with $S_{m}$, the Fourier transform of the $m$ th object image. The presence of these $N=2 M$ holograms, labeled $m=-M,-(M-1)$, $\ldots, 0, \ldots, M-1$, modulates the permittivity of the material such that the change in the permittivity of the medium can be written as

$$
\Delta \epsilon \approx \sum_{m=-M}^{M-1} R_{m}{ }^{*} S_{m}+\text { c.c. }
$$

where c.c. represents the complex conjugate term. ${ }^{6,7}$ In addition, using standard Fourier-optics analysis, ${ }^{9}$ one can express the $m$ th signal beam as

$$
\begin{aligned}
S_{m}(x, y, z) \approx & \exp [i(2 \pi / \lambda) z] \iint \mathrm{d} x_{o} \mathrm{~d} y_{o} f_{m}\left(x_{o}, y_{o}\right) \\
& \times \exp \left[-i(2 \pi / \lambda F)\left(x x_{o}+y y_{o}\right)\right] \\
& \times \exp \left[-i\left(\pi z / \lambda F^{2}\right)\left(x_{o}{ }^{2}+y_{o}^{2}\right)\right] .
\end{aligned}
$$

In the expression above, $f_{m}\left(x_{o}, y_{o}\right)$ is the $m$ th object image; $x, y$, and $z$ are the coordinates at the back focal plane of lens $\mathrm{L}_{1}$, and $F$ is the focal length of all three lenses $\mathrm{L}_{1}, \mathrm{~L}_{2}$, and $L_{3}$. By substituting relations (1) and (3) into relation (2), we can write out $\Delta \epsilon$ explicitly.

In readout, the recorded medium is illuminated with one of the set of phase-coded $\left(P_{j}^{n}\right)$ reference plane waves. Ideally, this would reconstruct only the hologram associated with that particular reference set. In what follows, we calculate the deviations of the reconstructed wave from the desired image. The amplitude of the diffracted plane-wave components, $E\left(\mathbf{k}_{d}\right)$, with wave vectors $\mathbf{k}_{d}$, can be derived from standard scalar diffraction theory. ${ }^{10}$ The following expression assumes that the Born and paraxial approximations are valid:

$$
E\left(\mathbf{k}_{d}\right) \approx \sum_{j=-M}^{M-1} \int \mathrm{d} \mathbf{r} P_{j}^{n} \exp [-i \mathbf{K} \cdot \mathbf{r} \Delta \epsilon(\mathbf{r})]
$$

where $\mathbf{K}=\mathbf{k}_{d}-\mathbf{k}_{j}, \mathbf{k}_{j}$ is the wave vector of one of the plane-wave components that make up an illuminating reference beam, $j$ is the readout pixel number corresponding to $\mathbf{k}_{j}$, and $n$ is the readout code-word number. 


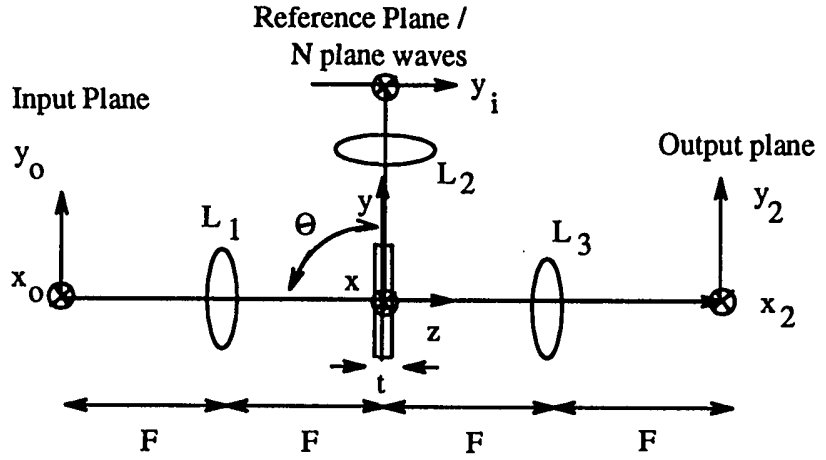

Fig. 1. Recording and readout geometry for $\phi$ multiplexing.

Lens $L_{3}$ maps each diffracted plane-wave component with wave vector $\mathbf{k}_{d}$ to a point $x_{2}, y_{2}$ at the output plane given by

$$
\mathbf{k}_{d}=\left[\frac{2 \pi x_{2}}{\lambda F}, \frac{2 \pi y_{2}}{\lambda F}, \frac{2 \pi}{\lambda}\left(1-\frac{x_{2}^{2}}{2 F^{2}}-\frac{y_{2}{ }^{2}}{2 F^{2}}\right)\right],
$$

assuming the paraxial approximation. Making use of the assumption that the transverse dimensions of the medium are much larger than the spatial bandwidth of the images, substituting relations (1)-(3) and (5) into relation (4), and carrying out the integration over the volume of the medium, we can write the electric field at the output plane as

$$
\begin{aligned}
E\left(x_{2}, y_{2}\right) \approx & \sum_{m=-M}^{M-1} \sum_{j=-M}^{M-1} \sum_{i=-M}^{M-1} P_{j}^{n} P_{i}^{* m} f_{m} \\
& \times\left(-x_{2}-\frac{F \lambda}{2 \pi} \Delta K_{i j x},-y_{2}-\frac{F \lambda}{2 \pi} \Delta K_{i j y}\right) \\
& \times \operatorname{sinc}\left\{\frac { t } { 2 \pi } \left[\Delta K_{i j z}+\frac{1}{F}\left(\Delta K_{i j x} x_{2}+\Delta K_{i j y} y_{2}\right)\right.\right. \\
& \left.\left.+\frac{\lambda}{4 \pi}\left(\Delta K_{i j x}{ }^{2}+\Delta K_{i j y}{ }^{2}\right)\right]\right\},
\end{aligned}
$$

where $\Delta \mathbf{K}_{i j}=\mathbf{k}_{i}-\mathbf{k}_{j}$ is the difference between the $i$ th reference wave vector $\mathbf{k}_{i}$ and the illuminating beam's wave vector $\mathbf{k}_{j}$. Also, $\Delta K_{i j \alpha}$ is the component of $\Delta \mathbf{K}_{i j}$ in the $\alpha$ direction. In relation (6), $t$ is the thickness of the material in the $z$ direction.

If $\Delta K_{i j \alpha}=0$ for all $i, j, \alpha$, then $E\left(x_{2}, y_{2}\right)=N f_{m}\left(-x_{2},-y_{2}\right)$, and we get our desired reconstruction. When $\Delta K_{i j \alpha} \neq 0$, cross-talk noise arises from the $m \neq n$ and the $m=n$ but $i \neq j$ terms in relation (6). We can write the components of $\Delta \mathbf{K}_{i j}$ in terms of the reference coordinates $y_{i}$ and $y_{j}$ :

$$
\begin{aligned}
\Delta K_{i j x} & =0, \\
\Delta K_{i j y} & =\frac{2 \pi}{\lambda F} \cos \theta\left(y_{j}-y_{i}\right)+\frac{\pi}{\lambda F^{2}} \sin \theta\left(y_{i}^{2}-y_{j}^{2}\right), \\
\Delta K_{i j z} & =\frac{2 \pi}{\lambda F} \sin \theta\left(y_{j}-y_{i}\right)+\frac{\pi}{\lambda F^{2}} \cos \theta\left(y_{j}{ }^{2}-y_{i}{ }^{2}\right),
\end{aligned}
$$

where $\theta$ is the angle between the normal to the reference plane and the optical axis of the input plane. To estimate the noise-to-signal ratio (NSR), we divide the total average noise power by the magnitude squared of the high signal level. We assume that each pixel of the stored images is an independent random variable that takes the values zero and one with equal probability. With use of the properties of Hadamard codes, the average NSR is given by

$$
\begin{aligned}
\mathrm{NSR}= & \frac{1}{N^{2}} \sum_{m} \mid \sum_{j} \sum_{i \neq j} P_{j}{ }^{n} P_{i}{ }^{m} \\
& \times\left.\operatorname{sinc}\left[\frac{t}{2 \pi}\left(\Delta K_{i j z}+\frac{\Delta K_{i j y} y_{2}}{F}+\frac{\lambda \Delta K_{i j y}{ }^{2}}{4 \pi}\right)\right]\right|^{2} .
\end{aligned}
$$

The spacing between the pixels of the phase code is chosen to be $\Delta y=(\lambda F / t \sin \theta) \equiv \Delta$. This makes the sinc function of Eq. (8) essentially zero at the center pixel $\left(y_{2}=0\right)$ of each hologram and hence minimizes cross talk at that location.

\section{RESULTS}

In order to calculate the NSR, we assume that $\theta=90^{\circ}$ to maximize capacity and that the reference pixels are spaced by $\Delta$. Rewriting Eq. (8) results in

$$
\begin{aligned}
\mathrm{NSR} & =\frac{1}{N^{2}} \sum_{m} \mid \sum_{j} \sum_{i \ngtr j} P_{j}{ }^{n} P_{i}{ }^{m} \\
& \times\left.\operatorname{sinc}\left[j-i+\frac{y_{2} \lambda}{2 F t}\left(i^{2}-j^{2}\right)+\frac{\lambda^{3}}{8 t^{3}}\left(i^{2}-j^{2}\right)^{2}\right]\right|^{2} .
\end{aligned}
$$

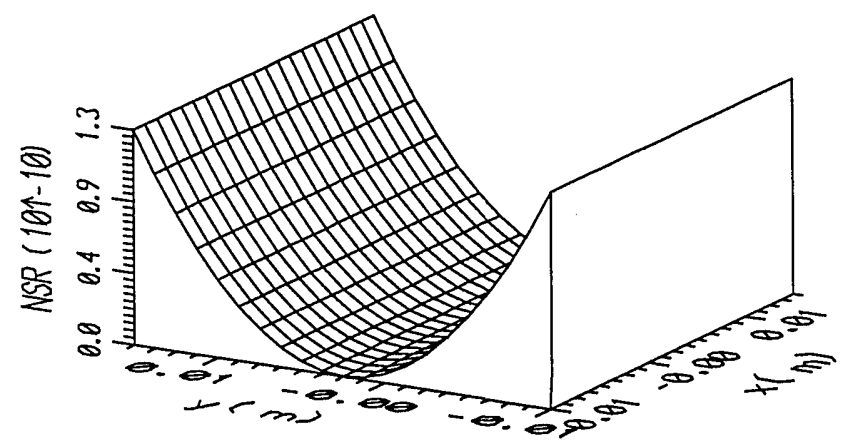

Fig. 2. NSR versus position $(x, y)$ on the output plane for the $n=8$ hologram, with $N=16$.

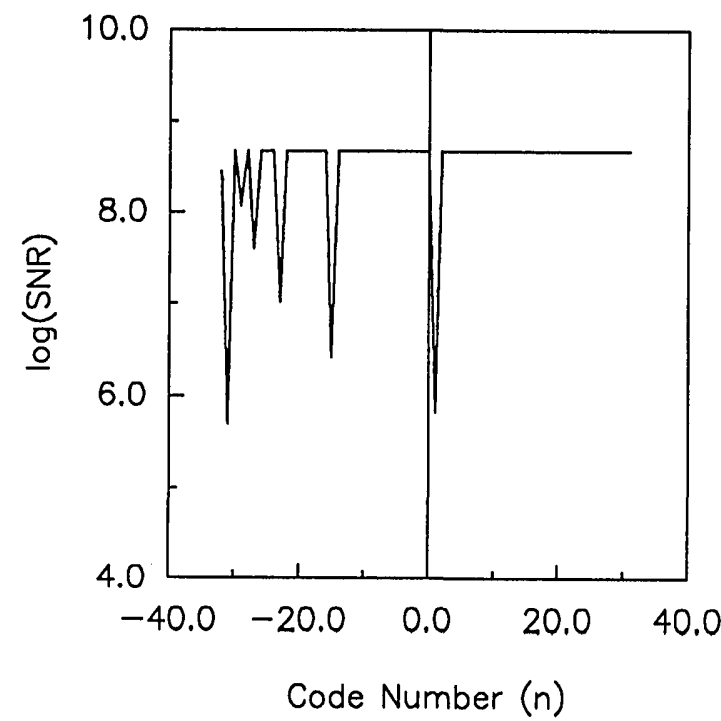

Fig. 3. $\log (\mathrm{SNR})$ versus hologram code number for $N=64$ holograms. 


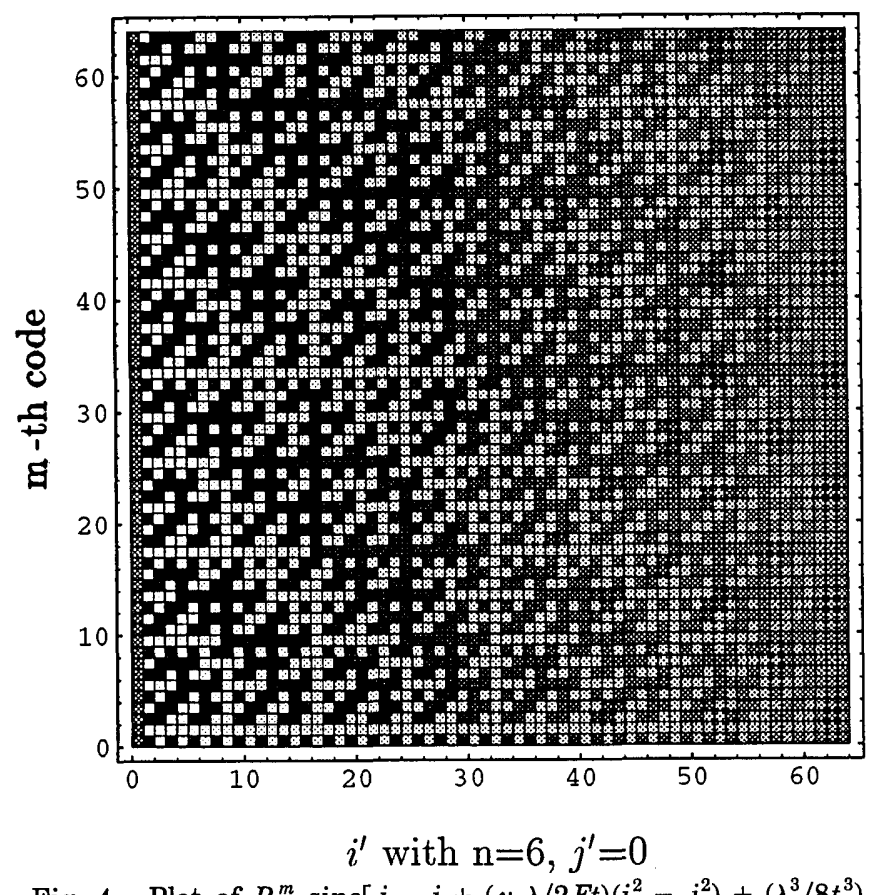
Fig. 4. Plot of $P_{i}^{m} \operatorname{sinc}\left[j-i+\left(y_{2} \lambda / 2 F t\right)\left(i^{2}-j^{2}\right)+\left(\lambda^{3} / 8 t^{3}\right)\right.$
$\left.\left(i^{2}-j^{2}\right)^{2}\right]$ as a function of $m$ and $i^{\prime}=i-M$ for $n=6$ and $j=0$.

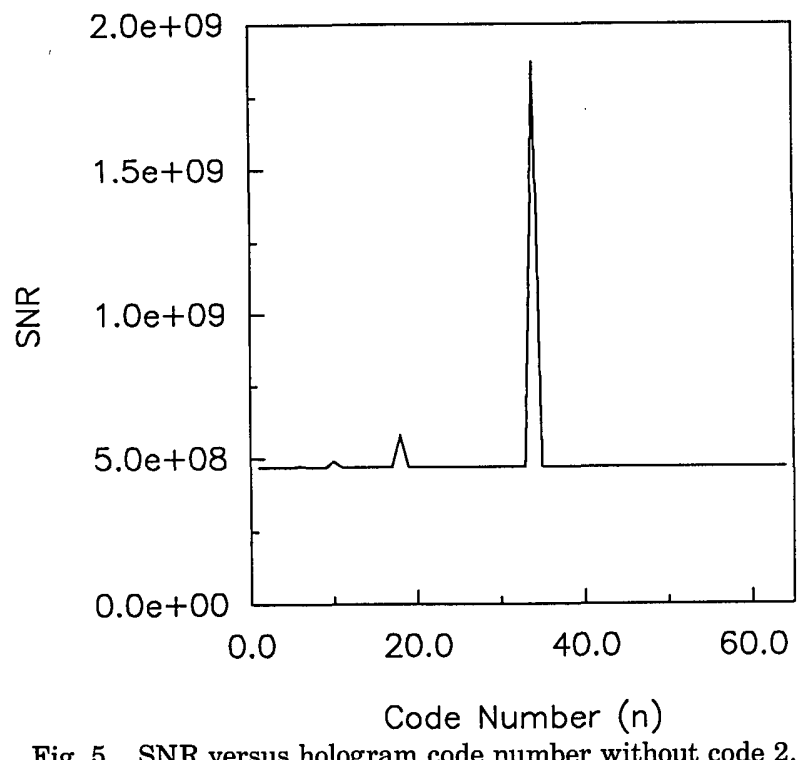

Fig. 5. SNR versus hologram code number without code 2 .

Notice that if the sinc functions are replaced by the Kronecker $\delta_{i j}$, then there is no cross talk. The NSR is a function of the location at the output plane in one dimension $\left(y_{2}\right)$. Given $t, \lambda, F, \theta$, and $N$, we can evaluate the above expression numerically. We calculated the NSR at the output plane for the $n=8$ hologram by numerically evaluating Eq. (9) with $F=30 \mathrm{~cm}, t=1 \mathrm{~cm}, x_{2 \max }=$ $y_{2 \max }=1.5 \mathrm{~cm}, \lambda=500 \mathrm{~nm}$, and $N=16$ holograms. Figure 2 shows the result plotted as a function of position on the output plane. Notice that the maximum NSR occurs at the $y_{2}$ boundary of the output plane.

Setting $y_{2}$ to its maximum value, we can plot the worstcase SNR versus $n$, the phase code number. Figure 3 shows the result for $N=64$ holograms. Code word num- ber 2 is the highest-frequency code word $(1,-1,1,-1 \ldots)$, and its structure complements the sinc function. Therefore when phase code number 2 is multiplied with the sinc function in Eq. (9), we obtain a consistently positive product that adds up to a large NSR. Figure 4 plots the product $P_{i}{ }^{m} \operatorname{sinc}\left[j-i+\left(y_{2} \lambda / 2 F t\right)\left(i^{2}-j^{2}\right)+\left(\lambda^{3} / 8 t^{3}\right)\right.$ $\left.\left(i^{2}-j^{2}\right)^{2}\right]$ as a function of $m$ and $i^{\prime}=i-M$ for $n=6$ and $j=0$. While this particular code word has a poor SNR, the others average to a much higher SNR. The other codes in Fig. 3 that have a poor SNR are noisy as a result of cross talk from code 2. Taking out code word number 2 , makes it possible to record holograms that have the same SNR (to a couple of decimal places), except for a few holograms that have better SNR than the others, as shown in Fig. 5. This result is different from $\theta$ multiplexing, ${ }^{6}$ in which most holograms have better SNR than the worst hologram, depending on their angular position with respect to the other holograms.

Figure 6 shows the worst-case SNR for $\phi$ and $\theta$ multiplexing with the same parameters as listed above. When using code word number 2 , the worst-case SNR decreases very quickly for $\phi$ multiplexing. Taking this code word out and recording $2^{n}-1$ holograms with $2^{n}$ plane-wave references results in a worst-case SNR that is approximately 3 orders of magnitude better than that of $\theta$ multiplexing. If we take the SNR for $\theta$ multiplexing at the maximum $y_{2}$, averaged over all holograms, we obtain an average SNR that is still more than two orders of magnitude less than that for $\phi$ multiplexing for large $N$. Therefore, well-chosen phase codes suppress, even on average, the cross talk. For $\lambda$ multiplexing, the worst-case SNR for 500 holograms, with all other parameters the same as in Fig. 6, is $1.4 \times 10^{3}$ (Ref. 7), compared with $10^{7}$ for $\phi$ multiplexing and $10^{4}$ for $\theta$ multiplexing. We have not calculated the cross talk for $\phi$ multiplexing beyond 512 holograms. Notice that the cross-talk SNR is very high in all cases, indicating that other system imperfections will most likely determine the fidelity of the reconstruction in practice.

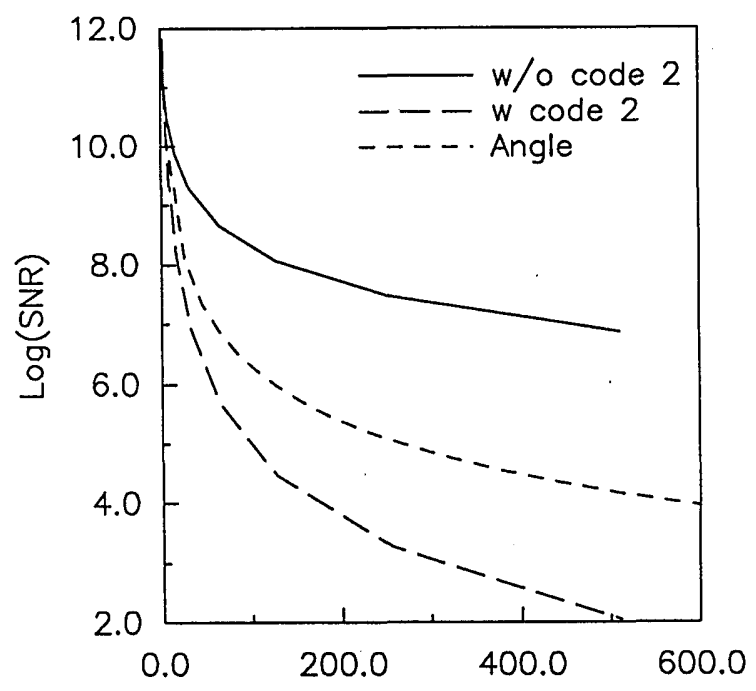

Number of Holograms

Fig. 6. SNR versus the total number of holograms for $\phi$ (with and without code 2 ) multiplexing and $\theta$ multiplexing. 


\section{ACKNOWLEDGMENTS}

Kevin Curtis is supported by a Northrop Fellowship. We thank the U.S. Air Force Office of Scientific Research for support of this work. In addition, we thank Geoffrey Burr, Sidney Li, and Fai Mok for their help.

\section{REFERENCES}

1. F. H. Mok, "Angle-multiplexed storage of 5000 holograms in lithium niobate," Opt. Lett. 18, 915-917 (1993).

2. G. A. Rakuljic, V. Leyva, and A. Yariv, "Optical data storage by using orthogonal wavelength-multiplexed volume holograms," Opt. Lett. 17, 1471-1473 (1992).

3. C. Denz, G. Pauliat, and G. Roosen, "Volume hologram multiplexing using a deterministic phase encoding method," Opt. Commun. 85, 171-176 (1991).

4. J. Trisnadi and S. Redfield, "Practical verification of holo- gram multiplexing without beam movement," in Photonics for Computers, Neural Networks, and Memories, S. T. Kowel, W. J. Miceli, and J. A. Neff, eds., Proc. Soc. Photo-Opt. Instrum. Eng. 1773, 362-371 (1992).

5. J. E. Ford, Y. Fainman, and S. H. Lee, "Array interconnection by phase-coded optical correlation," Opt. Lett. 15, 1088-1090 (1990).

6. C. Gu, J. Hong, I. McMichael, R. Saxena, and F. H. Mok, "Cross-talk-limited storage capacity of volume holographic memory," J. Opt. Soc. Am. A 9, 1978-1983 (1992).

7. K. Curtis, C. Gu, and D. Psaltis, "Cross talk in wavelengthmultiplexed holographic memories," Opt. Lett. 18, 1001-1003 (1993).

8. C. Denz, G. Pauliat, G. Roosen, and T. Tschudi, "Potentialities and limitations of hologram multiplexing by using the phaseencoding technique," Appl. Opt. 31, 5700-5705 (1992).

9. J. W. Goodman, Introduction to Fourier Optics (McGrawHill, New York, 1968), pp. 57-96.

10. J. D. Jackson, Classical Electrodynamics (Wiley, New York, 1975), pp. 427-432. 